\title{
Short-term production planning for district heating networks with JModelica.org
}

\author{
Stéphane Velut ${ }^{1}$, Per-Ola Larsson ${ }^{1}$, Johan Windahl ${ }^{1}$, Linn Saarinen ${ }^{2}$, Katarina Boman ${ }^{2}$ \\ ${ }^{1}$ Modelon AB, Ideon Science Park, SE-223 70 Lund, Sweden \\ ${ }^{2}$ Vattenfall R\&D, 81426 Älvkarleby, Sweden \\ stephane.velut@modelon.com, per-ola.larsson@modelon.com
}

\begin{abstract}
The short term thermal production planning problem is solved in two steps by integrating physical plant models into the standard approach. The first step aims at solving the discrete variables from the unit commitment sub-problem (UCP) using standard mixed integer linear models and optimization techniques. The second step focuses on the economic dispatch sub-problem (EDP) described by highfidelity, continuous time, physics-based Modelica models together with nonlinear optimization techniques from the JModelica.org platform. The output of the second step includes optimized power flows but also highly relevant variables such as supply temperature, supply flow rate, turbine by-pass valve in the cogeneration plant. The optimization is formulated as a maximization of the benefit from heat and electricity sell over a finite time-horizon.
\end{abstract}

The proposed method is validated in several test cases using experimental data from a plant in Nyköping. The optimizations demonstrate the feasibility and the high economic potential of the proposed approach when comparing with measurement data and the standard optimization techniques. The optimized planning schedules result in a balance between produced and consumed heat, priority to low-cost boilers and maximization plant revenue. Compared to measurement data, the optimizations result in a significantly lower supply temperature, a more extensive usage of the external cooler for higher efficiency and higher electricity production, fewer starts of units as well as an appropriate use of the accumulator tank.

The high-level description of optimization problems using JModelica.org provides useful means to speci- fy flexible optimization problems including constraints on arbitrary process variables such as heat load of the production units, supply temperature and flow rate, pressures.

Keywords: production planning; nonlinear optimization; district heating; physical modeling; unit commitment

\section{Introduction}

\subsection{Background}

\subsubsection{Production planning}

Production planning in district heating systems aims at finding a cost optimal scheduling of the heat and power production plants, which satisfies both the network load demand and operational constraints. Scheduling refers to the status of the production unit (on-off, a discrete variable), and the produced power (a continuous variable). The resulting optimization problem that involves both discrete and continuous variables is referred to as mixed integer non-linear problem (MINLP), for which no robust algorithm is available. It is therefore necessary to make reasonable assumptions on both the modeling and the computation approaches to get a tractable optimization problem. The problem can be described as being composed of two sub-problems:

- The Unit Commitment Problem (UCP), in which decisions are taken on whether a plant should be running or not. The main difficulty lies in the combinatorial nature of the problem.

- The Economic Dispatch Problem (EDP) in which the load decisions for all active plants are taken. The main difficulty lies in the nonlinearity of the plant. 
A good survey of the available approaches for shortterm production planning is given in [15].

As an input to the production planning problem, a predicted heat load over the entire optimization horizon should be provided. This is often generated by a load prediction model that typically includes a description of the district heating network and the effect of outdoor temperature. Here it is assumed that a perfect load prediction is available over the entire optimization horizon.

\subsubsection{Common approach}

The standard formulation of the thermal production planning problems relies on a simplified representation of the model equations. The plant models are typically linearized and the resulting problem becomes a Mixed Integer Linear Program (MILP). The continuous decision variables of the optimization problem are the energy flows whereas the influence of the supply temperature and mass flow are usually not modeled. This represents a limitation since e.g., supply temperature affects many critical parameters such as the amount of energy that can be stored in the network or the accumulator, the heat loss in the network and the electric efficiency of the cogeneration plants. To maintain a low model complexity, it is also common to describe all processes by static relationships except for the storage dynamics (heat and fuels) and eventually the transport delays in the distribution network. The linearization process, which is a necessary and critical part of the MILP approach, is consequently a trade-off between model accuracy and tractable model complexity.

\subsection{Proposed approach}

The proposed approach is based on the natural separation of the discrete problem (UCP) from the continuous one (EDP).

- UCP. The entire optimization problem is formulated using simple piecewise linear models and solved using a MILP solver. The main result of this stage is the status of every plant (on/off) over the optimization horizon.

- EDP. The desired load is dispatched between the running production plants to meet all plant operational and safety constraints. The status (on/off) of the plants and the start values of continuous control signals are given by the solution of the UCP.

The aim of the second step is to optimize the nonlinear plant model based on physical laws without any major simplification. The plants are described by mass and energy balances, in terms of enthalpy, mass flow rate and pressure. Dynamics can be included without restrictions to match the real dynamic behavior of the plants. The output of this second step includes optimized power flows, but also highly relevant variables that affect the production economy such as supply temperature, supply flow rate or turbine by-pass valve in the co-generation plant. This model complexity yields however a non-linear dynamic optimization problem and requires another type of solver than MILP solvers, see [1] for an overview of the available strategies. One reliable and efficient method to solve dynamic optimization problems that is based on non-linear programming solvers is the so-called collocation method. Control signals to be optimized and model equations are parameterized by a smaller number of variables, reducing considerably the complexity of the non-linear optimization problem. The original continuous-time optimization problem is transformed into a (discretetime) Non-linear Programming (NLP) problem that can be efficiently solved using commercial or opensource solvers. The authors have applied the collocation method for dynamic optimization of a Carbon Capture plant, see [2]. Other successful applications of this optimization technique have been reported in the literature, see [3] for a list of applications where IPOPT (Interior Point Optimizer), an open-source NLP solver, was used. To the authors' knowledge, limited work on applying large-scale NLP methods for solving the economic dispatch problem has been performed.

\section{Optimization tools and languages}

Two environments were used to define and solve the production planning problem:

- Dymola [9] was the chosen platform to derive, calibrate and simulate the physical Modelica [10] models defining the economic dispatch sub-problem

- Python was the chosen platform to solve both optimization problems and to do the post-processing

The unit commitment problem (UCP) was formulated in Python using the package PuLP [4] that is a light weight package that allows modelers to easily express mathematical programming problems, including mixed integer linear programs. It uses a high level modeling language and has been built to interface with commercial and open-source solvers. In the present work the solver CBC has been used, see [5]. 
The economic dispatch problem was solved from Python using the JModelica.org platform [16] as follows:

- The optimization problem was formulated in the Modelica and Optimica languages

- An FMU-simulation was carried out to generate a guess trajectory for the initialization of the nonlinear optimization

- The nonlinear optimization problem was solved using a collocation method available in JModelica.org

Applying nonlinear dynamic optimization techniques to solve the economic dispatch problem requires some considerations on initial trajectories, smooth media model functions, smoothing of mathematical functions such as absolute value, square root, min and max as well as scaling of optimization variables yielding a numerically sound behavior.

\section{Plant models}

\subsection{Idbäcken plant}

The Vattenfall $A B$ owned district heating plant Idbäcken, providing heat to customers in Nyköping, Sweden, served as reference plant. It contains in total seven different heat production units, one accumulator and one co-generation plant, see Figure 1. The Idbäcken plant has been the topic of interest in other research projects, see for instance [6] [7] and [8].

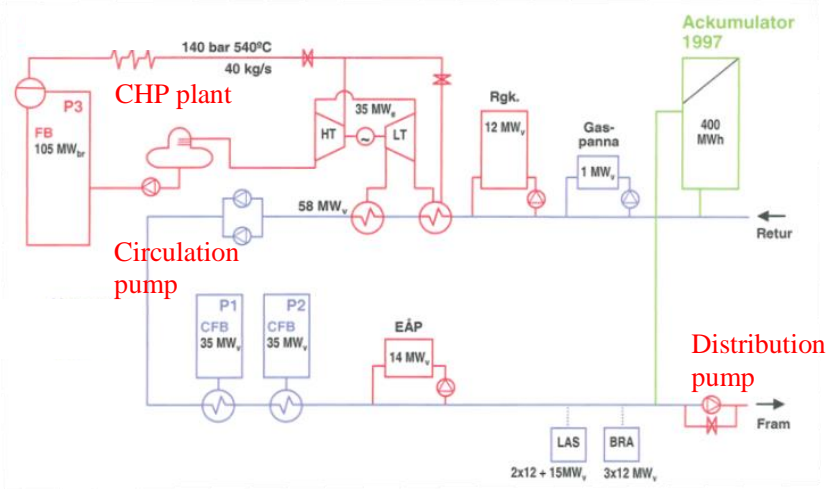

Figure 1. Schematic diagram of the Idbäcken plant with 7 heat production units, the accumulator and the co-generation plant

The main unit of Nyköping is the Combined Heat and Power plant (CHP) with a total heat capacity of $105 \mathrm{MW}$. The plant turbines can at most produce 35 MW of electricity, and the condensers can approximately transfer $75 \mathrm{MW}$ of heat to the district heat water. The flue gas of P3 is used in a flue gas con- denser of maximum $12 \mathrm{MW}$ to pre-heat district heat return water before entering the CHP plant.

Two circulating fluidized bed units, P1 and P2, of each $35 \mathrm{MW}$ used only for heat production may be used when heat demand is high. Additionally, there is an electric boiler of $14 \mathrm{MW}$ and two oil based heat production units at the hospital and the residential area Brandkärr with $2 \times 12$ and $3 \times 12 \mathrm{MW}$ production capacity, respectively.

There is also an accumulator tank of hot water and an external cooler Beriden (not shown in figure) that can be used to decrease the return temperature and therefore increase both the load and the efficiency of the co-generation plant for a higher electricity production.

\subsection{Models for UCP}

The unit commitment problem is formulated in Python using the PuLP modeling language.

\subsubsection{Heat production units}

The discrete time plant model, i.e., the UCP model, is very coarse compared to the EDP model. All heat producing units $i$, except for the CHP plant, are modeled only by the produced heat $Q_{i}$ and its minimum and maximum capacity. The influence of plant actuators such as pumps, valves or the influence of physical variables such as temperature, pressure and mass flows are not captured. The fuel consumption $U_{i}$ of a pure heat unit is calculated using the efficiency parameters $\eta_{i}$ as

$$
U_{i}=\frac{Q_{i}}{\eta_{i}}
$$

and for the CHP plant it is calculated as

$$
U_{3}=\frac{Q_{P 3}+P_{e l}}{\eta_{3}}
$$

For the CHP plant also the ratio between produced electricity and heat is interesting, defining the $\alpha$ value as,

$$
\alpha=\frac{P_{e l}}{Q_{P 3}}
$$

The accumulator energy $E_{a c c}$ is given by a simple integrator equation and is not temperature dependent:

$$
E_{a c c}[t]=E_{a c c}[t-1]-h Q_{a c c}[t-1]
$$

where $Q_{a c c}[t]$ is the energy flow from the accumulator, $h$ is the sample time and $t$ is the time index. 


\subsubsection{Co-generation plant $\mathrm{P} 3$}

The produced heat $Q_{P 3}$ and electric power $P_{e l}$ are influenced by the boiler load, the position of the turbine by-pass valve as well as the flow and temperature of the district water. It is by far too complex to describe this relationship by simple (piecewise) linear functions. By using a physics based Modelica plant model and varying the four main variables, a feasible region in the $Q_{P 3}-P_{e l}$-plane has been created to describe the behavior of the cogeneration plant. The resulting feasible region was described by four inequalities of the form

$$
a_{i} P_{e l}+b_{i} Q_{P 3}+c_{i} \geq 0
$$

where the points $a_{i}, b_{i}$ and $c_{i}$ are scalar coefficients.

The UCP optimization should optimize $Q_{P 3}$ and $P_{e l}$ with the constraint that they should be in the feasible region described by the inequalities.

\subsection{Models for EDP}

The models used to formulate the economic dispatch problem are implemented in Modelica.

\subsubsection{Medium model}

Two different types of media models are used in the model package:

1. Simple water media - a water media with constant specific heat capacity and density, used to describe the water in the district heating network.

2. Advanced water media - a water media with polynomial functions approximating IF97 reference functions, used to describe liquid and vapor water in the cogeneration plant, see [17] for more details.

\subsubsection{Cogeneration plant}

The co-generation (heat and electricity production) unit P3, shown in Figure 2, is described by the following models

- One high pressure turbine and two low pressure turbines, all with bleed streams

- Two condensers

- By-pass valve for by-passing steam directly to the condensers

- A lumped bleed streams to describe the flow to the de-aerator and the high-pressure preheater

- Control volumes
The characteristics that are important to capture with respect to the optimization is the influence of the turbine by-pass valve, the district heat network flow and temperature and the boiler load on the produced heat and electricity. It is not necessary to describe e.g., the furnace, instead, focus has been directed towards the vapor cycle. The main modeling simplifications on the vapor cycle is that it is not closed, which results in the following assumptions:

- The vapor characteristics (pressure and enthalpy) at the boiler outlet are constant and the boiler load linearly affects the boiler mass flow rate.

- The feed water heaters that are downstream of the condensers are not modeled. All the bleed streams that normally go to the nonmodeled pre-heaters are represented by a single stream connected to a constant pressure source.

- The condensate leaving the condenser is assumed to be at saturation pressure.

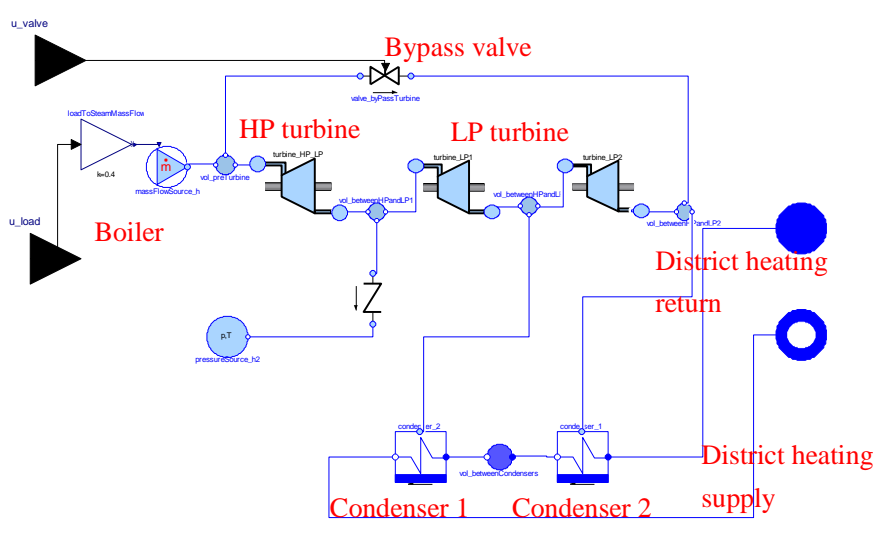

Figure 2. Dymola model of the cogeneration plant P3 with connections to the district water.

\subsubsection{Fluid component models}

\subsubsection{Turbine}

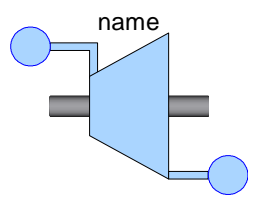

Physics-based model defined by an isentropic efficiency to calculate the outlet enthalpy and turbine work. The mechanical power generated from the steam is calculated using a mechanical efficiency and the pressure drop is related to the flow rate using Stodola's law. The electric power is calculated from the mechanical power using an efficiency parameter (generator losses). 


\subsubsection{Condenser}

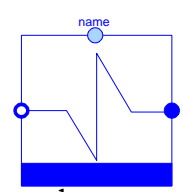

The heat flow rate transferred to the district water is driven by the temperature difference between the incoming water and the saturation temperature in the condenser. This heat flow rate is further used to compute the condensation rate that drives the bleeding flow from the turbine.

\subsubsection{Control Volume}

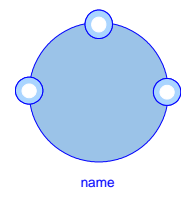

The control volume is a straightforward implementation of dynamic mass and energy balances expressed using pressure and enthalpy as states. Temperature is computed using pressure and enthalpy. The model requires partial derivatives of density with respect to enthalpy and pressure.

\subsubsection{Valve and pressure loss}

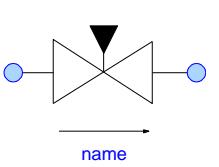

The mass flow through the valve is computed using the pressure difference, the valve opening and data from a nominal point. A standard quadratic equation relates mass flow and pressure drop.

\subsubsection{Circulating Fluidized Beds, P1 and P2}

$\checkmark{ }_{P 1}$ The model is not physics-based and heat CFB transferred to the water is calculated by firstorder filtering of the load and using paramePors for efficiency and maximum heat transfer.

\subsubsection{Electric Boiler and Oil Driven Boilers}

$\nabla$ The model is not physics-based and heat transferred to the water is calculated by the load and using parameters for efficiency and maximum heat transfer.

\subsubsection{Accumulator}

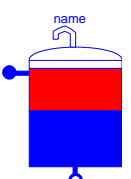

The accumulator is modeled using a finitevolume approximation that neglects buoyancy effects, i.e. no mixing is assumed when the accumulator is not charging or discharging. The accumulator is charged and discharged from the top and bottom. Return water enters from the bottom. Heat loss has been neglected.

\subsubsection{External cooler}

The cooler is modeled as a lumped epsilon-NTU heat

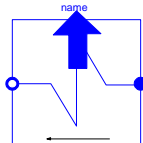
exchanger where heat transfer is driven by the difference between the inlet temperatures. It is assumed that the minimal heat capacity flow is always on the district side

flow.

\subsubsection{Transport Pipe}

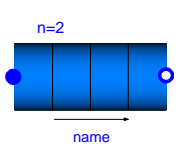

It is a finite volume implementation of a pipe with control volumes in series. The nominal number of discretization segments is 4 and the pipe diameter and length are parameters.

\subsubsection{Flue Gas Condenser}

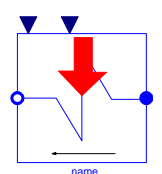
The model is not physics-based and is an ideal heat source which produces constant heat as long as P3 is running.

\subsubsection{Pump}

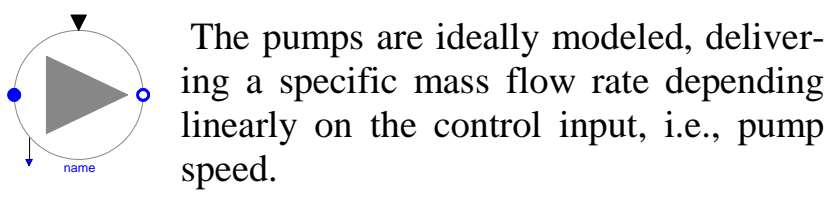

\subsubsection{Splitter/Valve}

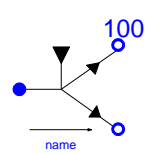

The splitter/valve is ideally modeled and splits the incoming flow into two flows which sizes depend on the control signal to the splitter.

\subsubsection{Customer}

name The customer is modelled with a fixed outlet temperature and the heat absorbed $\because \quad$ is calculated by the temperature difference between in- and outlet and the flow that is merely propagated through from in- to outlet.

\section{Problem formulation}

\subsection{Degrees of Freedom}

The discrete time optimization (UCP) includes the power heat flows, the electricity production rate as well as the status (on/off) of every unit as optimiza- 
tion variables. The status of each unit is fixed in the continuous optimization.

The continuous time optimization problem contains 11 degrees of freedom, namely:

- $U_{i}(t)$ fuel load of production unit $i, 1 \leq i \leq$ 6

- $U_{B P}(t)$, turbine by-pass valve position

- $U_{C P}(t)$, Speed of circulation pump

- $U_{D P}(t)$, Speed of distribution pump

- $U_{\text {split }}(t)$, ratio between flows going to customer and by-passing customer respectively

- $U_{\text {cooler }}(t)$, ratio between flows going to external cooler (Beriden) and by-passing it

In the optimizations however, the decision variables used by the optimization routine will be the derivatives of the above inputs. Thus, an equation on the form

$$
U_{j}(t)=\int_{t} \dot{U}_{J}(t) d t
$$

is introduced for each input. This extension makes it easy to set minimum and maximum constraints on the input signal derivatives in the optimizations. In Modelica code, for a general input $u$ with minimum and maximum values $u_{\text {min }}$ and $u_{\max }$ and minimum and maximum derivative values $\dot{u}_{\min }$ and $\dot{u}_{\max }$, an example is the following:

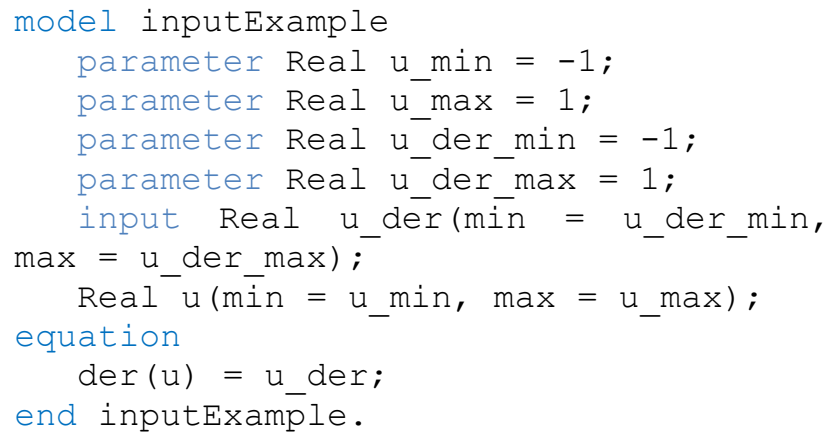

Another possibility is to utilize the constraint-section in the optimization model.

\subsection{Cost function}

The goal of the optimization, both in the UCP and EDP, is to produce enough heat to follow the customer heat load over time and at the same time do it as economically beneficial as possible. The EDP problem considers fuel costs $U_{i}(t) p_{i}$ and incomes from selling heat $P_{e l}(t) p_{e l}$ and electricity $Q(t) p_{Q}$ to describe the plant economy. Note that operational costs for e.g., pumps are not considered. The cost for starting a production unit $s t a r t_{i}[t] s_{i}$ is only included in the objective function of the UCP sub-problem. Hence, the revenue at a time instant can thus be formulated as

$$
\begin{aligned}
R(t)=P_{e l}(t) p_{e l} & +Q(t) p_{Q} \\
& -\sum_{i} U_{i}(t) p_{i}-\operatorname{start}_{i}(t) s_{i},
\end{aligned}
$$

A minor cost on the input derivatives must be used for regularity reasons. This cost at a certain time instant is formulated as

$$
\dot{W}(t)=\sum_{\substack{j=\text { All model } \\ \text { inputs }}} q_{\dot{U}_{j}} \dot{U}_{j}(t) .
$$

where $q_{\dot{U}_{j}}$ is the weight for derivative $\dot{U}_{j}$.

The cost function to be minimized, considering the cumulative revenue over the optimization horizon, can thus be formulated as

$$
J=\int_{t_{0}}^{t_{f}}(\dot{W}(t)-R(t)) d t
$$

where the optimization interval is $\left[t_{0}, t_{f}\right]$ and $24 \mathrm{~h}$ long. This is as long as the optimization horizon in the UCP and also as the prediction time series provided by the heat load prediction model. Without the extra term of the input derivatives, this cost function is the continuous time counter part of the cost function in the UCP except for the start-up costs.

\subsection{Constraints}

\subsubsection{Common constraints for EDP/UCP}

For fulfillment of the heat demand from the customers, the following constraint is used in both EDP and UCP:

$$
-Q_{\text {dev.max }} \leq Q(t)-Q_{d}(t) \leq Q_{\text {dev.max }}
$$

where $Q$ is the heat delivered to the customers, $Q_{d}$ the desired heat load from the prediction model. The maximum deviation $Q_{\text {dev.max }}$ is set to $1 \mathrm{MW}$. The supply temperature is not a direct function of the outdoor temperature, which is common practice. Instead, the supply temperature depends on the supply flow and heat demand, where the latter is a prediction depending on predicted outdoor temperature. A lower bound on the supply temperature has also been introduced and this could be replaced by a lower bound on the temperature at the customer substation if the district heating network is modelled. 
An accumulator energy end-point constraint is introduced in both EDP and UCP to avoid that the accumulator gets empty at the end of the optimization interval:

$$
E_{a c c}\left(t_{f}\right) \geq E_{a c c}\left(t_{0}\right)
$$

The input derivatives, i.e., the decision variables for the optimizer, are given minimum and maximum values. A maximum change of $\pm 2 \% / \mathrm{min}$. has been set for all input derivatives relative to the maximum value of the input to the model. These limits can be set both from a physical perspective but also from a numerical perspective such that highly changing control signals are avoided. In the optimization results presented in this report, the derivative constraints are far from being active.

\subsubsection{UCP specific constraints}

The start and stop of large solid fuel boilers is timeconsuming and it is therefore critical to model the delay between the start/stop decision and the time the boiler is running at minimum/zero load. All units that are starting or stopping are required to follow a pre-defined trajectory $Q_{i, s t a r t}[t]$ or $Q_{i, s t o p}[t]$, respectively. The constraints are formulated as

$$
\begin{aligned}
Q_{i}[t] & =Q_{i, \text { start }}[t], \\
t & \in\left[t_{\text {start }}, t_{\text {start }}+t_{\text {startdelay }}\right] \\
Q_{i}[t] & =Q_{i, \text { stop }}[t], \\
t & \in\left[t_{\text {stop }}, t_{\text {stop }}+t_{\text {stopdelay }}\right]
\end{aligned}
$$

where $t_{\text {startdelay }}$ and $t_{\text {stopdelay }}$ are the lengths of start and stop sequences in time. The details on how the timing variables $\operatorname{start}_{i}[t], \operatorname{stop}_{i}[t]$, starting $_{i}[t]$ and stopping $i_{i}[t]$ relate to the starting and stopping sequence equations above can be found in [11].

The accumulator storage capacity and heat flow are constrained with minimum and maximum values as

$$
\begin{gathered}
E_{a c c, \min } \leq E_{a c c}[t] \leq E_{a c c, \text { max }} \\
Q_{a c c, \min } \leq Q_{a c c}[t] \leq Q_{a c c, \max } .
\end{gathered}
$$

\subsubsection{EDP specific constraint}

Normally, the supply temperature is chosen as a function of the outdoor temperature. In the continuous optimization, the supply temperature is optimized and allowed to vary between $74.5^{\circ} \mathrm{C}$ and $110^{\circ} \mathrm{C}$. Also the flow to the customers, essentially the distribution pump flow, is given minimum and maximum values. These are $0 \mathrm{~kg} / \mathrm{s}$ and $550 \mathrm{~kg} / \mathrm{s}$.

\section{Optimization example}

Different test cases based on measurement data from Idbäcken plant were considered to evaluate the production planning strategy. All optimizations, both UCP and EDP, are performed using an optimization horizon of $24 \mathrm{~h}$. The UCP sampling time is $0.5 \mathrm{~h}$ while the number of elements in the collocation scheme is 72 , i.e., the length of each element is 20 minutes. One test case with two load peaks is presented in the paper.

\subsection{Initialization}

All dynamic models were initialized using experimental data of the real plant. The variables of the UCP model that require initialization are the status of each unit, the heat and electricity production of the co-generation plant as well as the accumulator energy. The physical model of the plant used to formulate the EDP sub-problem contains much more states to be initialized. The start values were either directly taken from the measurement data or computed using an FMU simulation of the plant.

\subsection{Computational statistics}

In all optimization cases, the UCP optimization formulation results in a MILP of approximately 4000 decision variables and 7000 constraints. The sampling time considered is 30 minutes and the optimization horizon is $24 \mathrm{~h}$. With the CBC MILP solver, see [5], the solution time is less than $25 \mathrm{~s}$ for all optimization cases.

The resulting NLP, after discretization of the EDP optimization problem using 72 elements each of a length 20 minutes, is solved using IPOPT v3.10.0 running with the linear solver MA27, see [12] and [13]. The NLP contains approximately 130000 variables for each of the considered optimization cases, which can be considered a small to medium sized problem for IPOPT. Much larger problems have been solved using the JModelica.org framework and IPOPT, see [14] and references therein. The solution time is approximately 2-5 minutes and depends on e.g., initial guesses number of decision variables and number of active constraints.

\subsection{Specific test case}

\subsubsection{Experimental data}

In this test case, the co-generation plant P3 is the only running unit at the beginning of the considered 
time interval. The heat load profile showed in the figure below displays two peaks that require the start of additional units. To meet that increasing customer demand, the heat production in the cogeneration plant was first prioritized by fully opening the bypass valve before both $\mathrm{P} 1$ and $\mathrm{P} 2$ were started, see dotted curves in Figures 4 and 5.

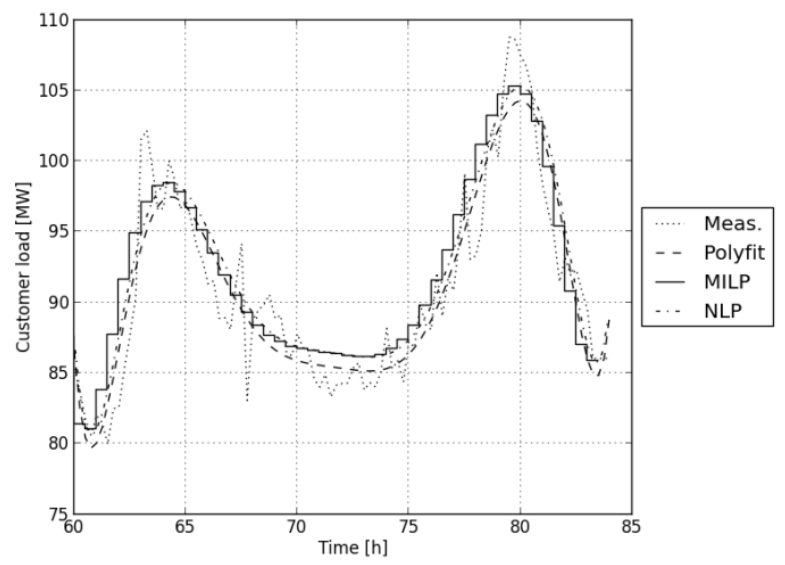

Figure 3. Profile of the customer heat load: measurement, polynomial approximation, MILP and NLP solutions.

\subsubsection{Optimization results}

The solution of the unit commitment problem is characterized by the start of $\mathrm{P} 2$ at the first sample to meet the increasing customer demand. The start of P1 does not seem to be necessary. As the first peak of the customer heat demand comes before P2 is fully available, the accumulator is used and its energy level is significantly decreased during the first half of the optimization interval. P2 stays at its minimum capacity during the first $12 \mathrm{~h}$, a stop and re-start would be a more expensive alternative. During the second half of the optimization, P2 is used to load the accumulator and provide heat to the network. The optimization leads to a constant and maximum heat electricity production as it is economically beneficial. The turbine by-pass valve is therefore kept closed during the entire optimization interval. The variation in the customer load is met by changing the load in P2 and by using the accumulator. Figure 6 shows one typical advantage of applying nonlinear optimization for the economic dispatch: the supply temperature and flow are optimized to maximize the benefits. Note that none of these variables are in the UCP formulation as the UCP only contains heat and energy variables. For minimal fuel consumption, the supply temperature is kept low, about 5 degrees lower than in measurement data, the flow rate to the network is instead increased to meet the heat power demand. Note that the distribution pump operates close to its maximal capacity except when the accumulator needs to be charged. Accumulator loading requires indeed a difference in the flows delivered by the circulation and the distribution pumps. At $\mathrm{t}=70 \mathrm{~h}$, the circulation pump operates at its maximum and the distribution pump is therefore forced to be decreased for accumulator loading.

\subsection{Conclusion from other test cases}

The production planning strategy has been successfully tested and compared with experimental data in 6 different cases, see [18] for more details. The following conclusions can be drawn:

- It is fully possible to integrate nonlinear optimization techniques from JModelica.org into the standard production planning approach for more accurate and more informative production plans.

- One of the main advantage of combining physical modeling and nonlinear optimization techniques is the optimized supply temperature and mass flow rate as well as a more accurate description of the accumulator.

- In the considered network, the optimization showed a high potential income related to the use of the external cooler that reduces the return temperature for a higher electric efficiency and a higher electricity production. This only occurs at low customer loads, i.e., when P3 is not required to run on full load. If customer load is high, then P3 should already be running at full load and the electricity production maximized.

- Handling of constraints on physical variables such as temperature, pump capacity and also constraints related to the way the accumulator is connected, improves substantially the quality of the optimization results.

- Supply temperature can be decreased by approximately $4^{\circ} \mathrm{C}$ compared to experimental data. With a perfect load prediction, the savings compared to measurement data is about $8 \%$, which is related to the extensive use of the external cooler. 

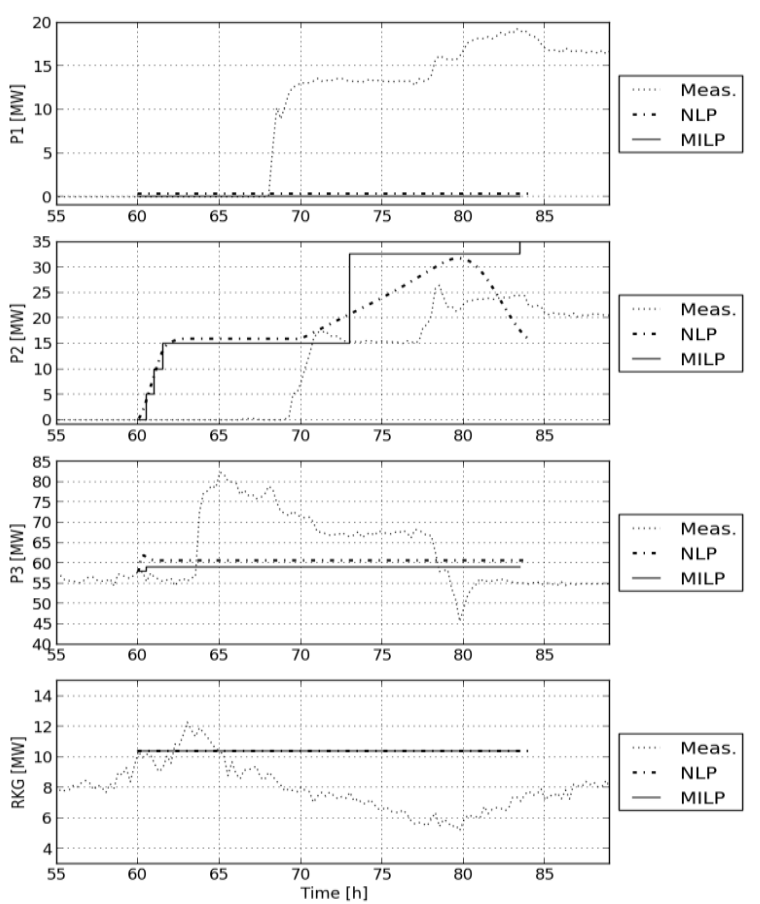

Figure 4. Heat production of the main units: measurement (dotted), MILP (solid) and NLP (dashed).
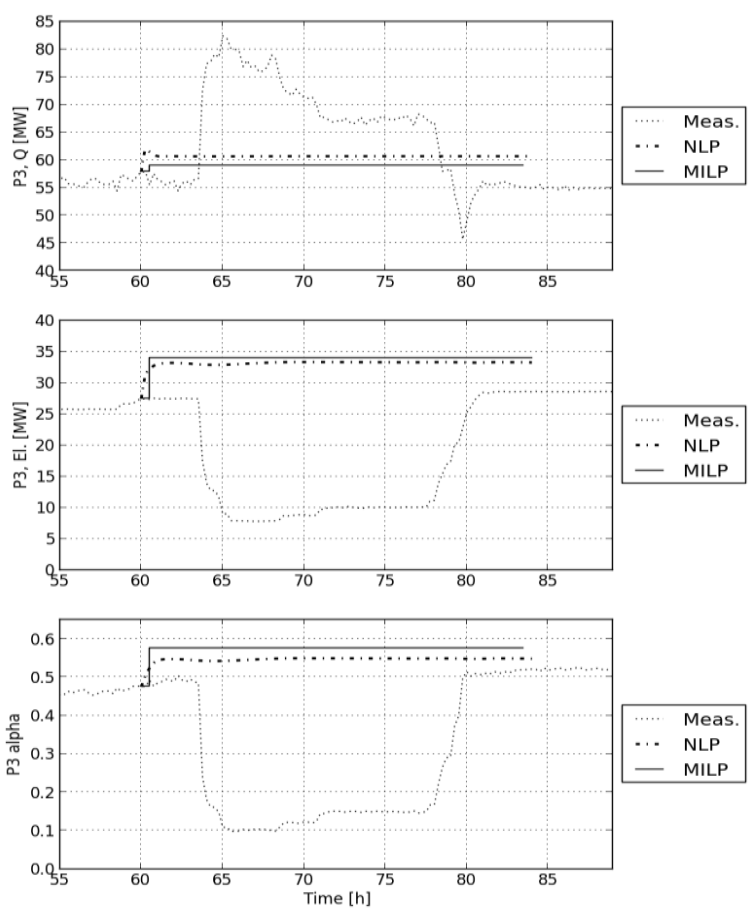

Figure 5. Heat, electricity production rates and alphavalue of the cogeneration plant $P 3$.
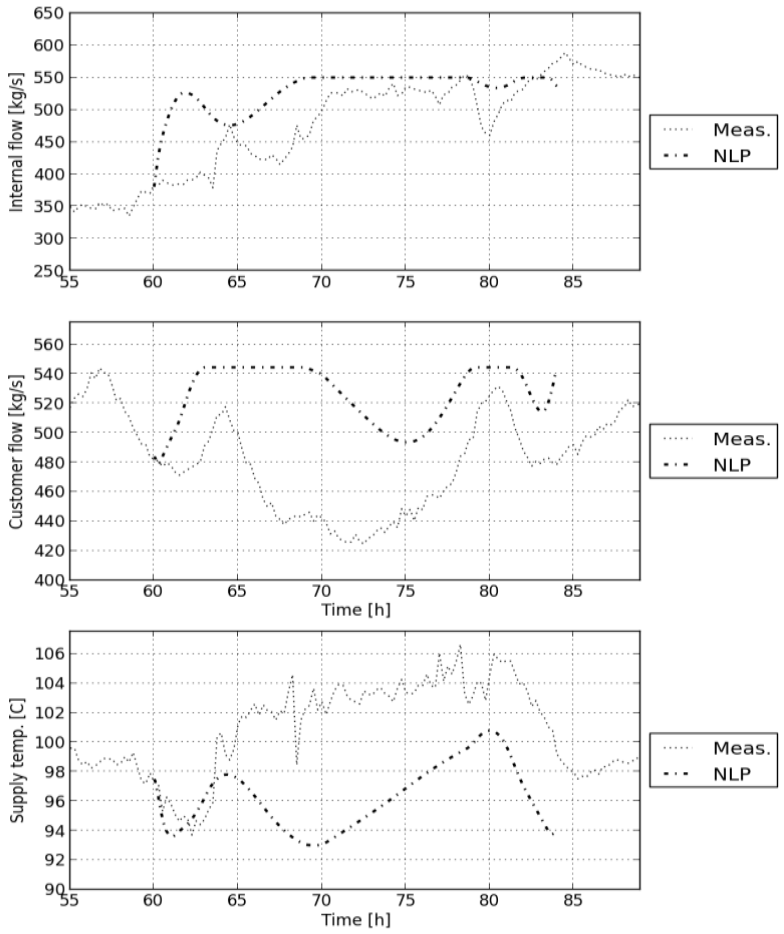

Figure 6. Circulation flow, flow to network and supply temperature. Measurement (dotted) and NLP (dashed).
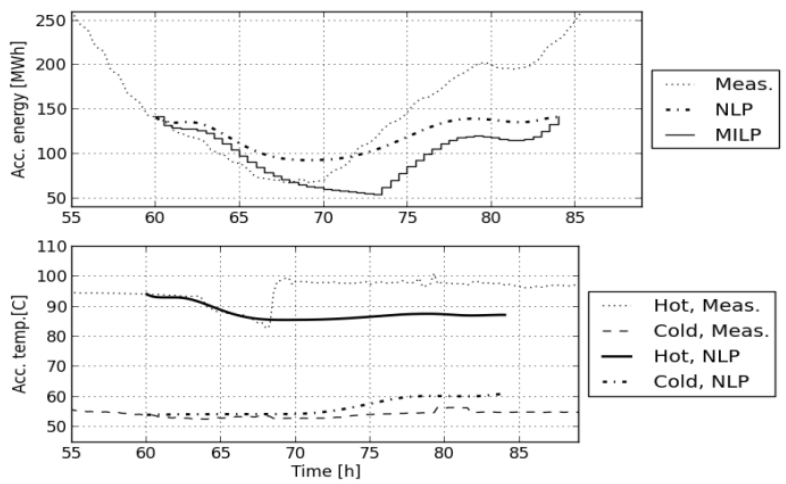

Figure 7. Accumulator energy and temperatures.

\section{Conclusions}

A method that substantially improves the standard approach for short-term production planning has been proposed in the paper. It is based on the use of physical plant models and nonlinear optimization techniques from JModelica.org to solve the economic dispatch sub-problem. The strategy has been validated using experimental data from a plant in Nykö- 
ping Sweden. Compared to measurement data, the method results in a significantly lower supply temperature, a more extensive usage of the external cooler for higher efficiency and higher electricity production. The flexible optimization platform JModelica.org makes it possible to optimize the plant economy and introduce constraints on critical variables such as temperature, pressure or flow.

\section{Acknowledgement}

Värmeforsk, "The Swedish Thermal Engineering Research Institute" and Energimyndigheten "The Swedish Energy Agency" are gratefully acknowledged for their financial support (project P12-203).

\section{References}

[1] C. Cervantes and L. T. Biegler, "Optimization strategies for dynamic systems," in C. Floudas, P. Pardalos (Eds), Encyclopedia of Optimization, 2000.

[2] J. Åkesson, C. Laird, G. Lavedan, K. Prölss, H. Tummesheit, S. Velut and Y. Zhu, "Nonlinear Model Predictive Control of a $\mathrm{CO} 2$ post-combustion unit," Chemical Engineerging Technology, 2011.

[3] Ipopt homepage, coin-or, http://projects.coinor.org/Ipopt/wiki/IpoptPapers.

[4] S. Mitchell, A. Mason, M. O'Sullivan and A. Phillips, "PuLP: a linear programming toolkit for python," http://www.coin-or.org/PuLP/.

[5] CBC Team, "CBC home page," 2013. [Online]. Available: https://projects.coinor.org/Cbc. [Accessed 12 August 2013].

[6] L. Saarinen, "Model-based control of district heating supply temperature," Värmeforsk P08-819, 2010.

[7] L. Saarinen and K. Boman, "Optimized district heating supply temperature for large networks," Värmeforsk P08-830, 2012.

[8] L. Saarinen, "Modeling and control of a district heating system," Master thesis, Uppsala University, 2008.

[9] Dassault Systemes, "Dassault Systemes Home Page," 2013. [Online]. Available: http://www.3ds.com/products/catia/portfolio/ dymola. [Accessed 6 August 2013].
[10] The Modelica Association, "The Modelica Association Home Page," 2013. [Online]. Available: http://www.modelica.org. [Accessed 6 August 2013].

[11] J. Arroyo and A. Conejo, "Modeling of startup and shut-down power trajectories of thermal units," IEEE Transactions on power systems, vol. 19, no. 3, 2004.

[12] A. Wächter and L. T. Biegler, "On the implementation of an interior-point filter line-search algorithm for large-scale nonlinear programming," Mathematical Programming, vol. 196, no. 1, pp. 25-68, 2006.

[13] HSL, "A collection of Fortran codes for large scale scientific computation," 2013. [Online]. Available: http://www.hsl.rl.ac.uk. [Accessed 13 August 2013].

[14] P.-O. Larsson, PhD thesis: Optimization of Low-Level Controllers and High-Level Polymer Grade Changes, Lund, 2011.

[15] E. Dotzauer, "Algorithms for Short-Term Production planning of Cogeneration Plant," Lic. Thesis, Linköping University, 1997.

[16] "www.jmodelica.org," Modelon AB, 2013. [Online]. Available: www.jmodelica.org. [Accessed 2013].

[17] Bauer, O. Modelling of Two-Phase Flows with Modelica, Master's Thesis, Lund University, Department of Automatic Control, 1999.

[18] S. Velut, P.O. Larsson, J. Windahl, L. Saarinen, K. Boman, Non-linear and Dynamic Optimization for Short-term Production Planning. Värmeforsk report, 2013. 